\title{
Evolution of geomagnetic cutoffs at the South Pole and neutron monitor rates
}

\author{
Laura Rose Rosen* \\ Washington State University \\ E-mail: laura.rosen@wsu.edu

\section{Surujhdeo Seunarine} \\ University of Wisconsin-River Falls \\ E-mail: surujhdeo. seunarine@uwrf.edu
}

\begin{abstract}
Neutron monitors are among the most robust and reliable detectors of $\mathrm{GeV}$ cosmic rays and are sensitive, with high precision, to modulations in Galactic Cosmic Rays (GCR). Hence, they can be deployed for extended periods of time, decades, and are able to observe the modulation of GCR over many solar cycles. The South Pole Neutron Monitor, located at the geographic South Pole, which is both high latitude and high altitude, has an atmospheric cutoff of around $0.1 \mathrm{GV}$. In the first four decades of its operation, a secular decline in the neutron rates have been observed. The decline may have leveled off recently. Environmental effects, including snow build up around the housing platform and the emergence and relocation of structures at the South Pole have been ruled out as causes of the decline. A recent study challenged the assumption that geomagnetic effects can be ignored at the South Pole, in particular for cosmic rays approaching from select regions in azimuth and at large zenith angles. This work confirms that ignoring geomagnetic cutoff effects could be important for the South Pole Neutron Monitor rates. We extend the investigation to include particle propagation in the Polar atmosphere, and the evolution of cosmic ray cutoffs at the South Pole over several decades. A connection is made between the evolving cutoffs and the decline in neutron monitor rates.
\end{abstract}

36th International Cosmic Ray Conference -ICRC2019-

July 24th - August 1st, 2019

Madison, WI, U.S.A.

\footnotetext{
*Presenting Author.
} 


\section{Apparent Decline in South Pole Neutron Monitor Rates}

Cosmic rays can be observed indirectly by neutron monitors [1] on the earth's surface. The detector component of a neutron monitor is a proportional counter containing either ${ }^{3} \mathrm{He}$ or $\mathrm{BF}_{3}$ gas. Depending on the design, the central counter is surrounded by layers of paraffin wax or polyethylene, which acts to reflect environmental neutrons or moderate the energy of cosmic ray secondary neutrons. A layer of lead acts as a producer in which a nuclear reaction between the lead and neutrons result in the production of evaporation neutrons, which are the neutrons observed in the proportional counter. Modulation on different time scales are readily observed, the dominant one being the 11-year periodicity in intensity associated with the reversal in polarity of the sun. The neutron monitor at the Amundsen-Scott South Pole Station has operated since 1964, save for two brief periods when it was shut down. It sits on the glacial ice at the South Pole at an altitude of $2820 \mathrm{~m}$. The effective vertical cutoff rigidity is assumed to be constant at $0.1 \mathrm{GV}$. Of the dozens of neutron monitor stations operating around the world, the South Pole Neutron Monitor (SOPO) is the only one that has experienced an apparent steady decline in its count rate for over five decades [2] [3] [4]. In the years since SOPO has been operating, the monitor type has changed and it has been relocated at the Pole. A detailed investigation has ruled out these changes [4] as the cause of the decline. Snow accumulation and structures have been accounted for. [3] showed that the evolution of geomagnetic cutoffs for detection at the South Pole, as well as particle propagation, may be important in explaining the decline. We continue to study the decline by carrying out detailed simulations of geomagnetic fields for the past five decades, and include

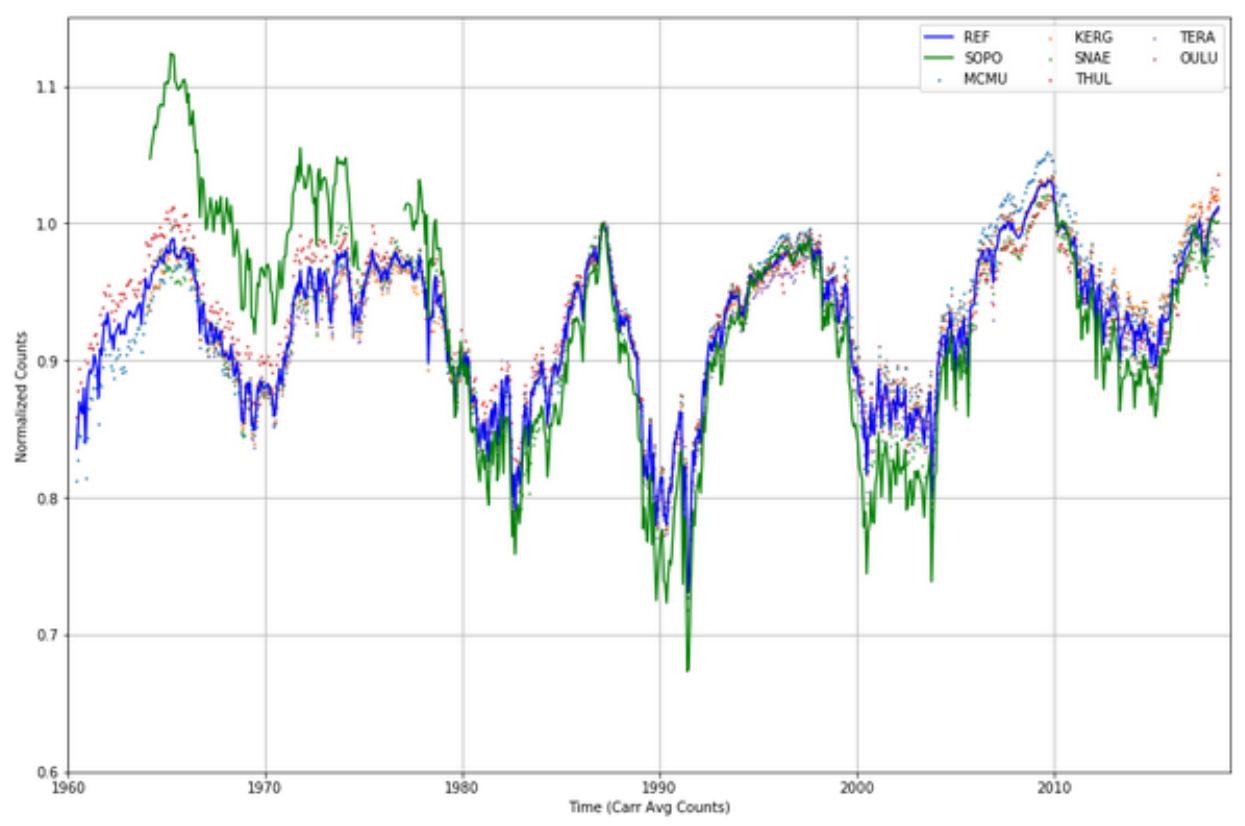

Figure 1: Normalized pressure and efficiency corrected rates of several lower rigidity cutoff neutron monitors: South Pole(SOPO), McMurdo(MCMU), Kerguelen(KERG), Sanae(SNAE), Thule(THUL), Terre Adelie(TERA), and Oulu(OULU). The blue reference curve is found by averaging the rates of all stations except SOPO's. The rates are normalized to the peak rate in 1987. 
a study of particle propagation in the atmosphere. Figure 1 shows what is being investigated. Data, corrected for pressure and efficiency, for several low cutoff neutron monitors are shown. For convenience of presentation, data are normalized to the peak intensity in 1987 . The 11 year solar cycle is evident, but there is an approximate 10\% decrease in SOPO's rates over the first few decades. Past 2000, it appears that the decline in rates is not as strong as in earlier years, but the rates are still systematically lower when compared to other monitors.

\section{Simulations}

MAGNETOCOSMICS [5] is a GEANT4 [6] based particle tracking code for the Earth's magnetosphere. It allows one to compute the trajectory of charged particles in two geomagnetic field models: IGRF, Tsyganenko. The magnetic field models are configurable, allowing the simulation of fields for selected years. Thus, one can simulate the evolution of rigidity cutoffs and asymptotic directions, in particular for the location of SOPO. When calculating the cutoff rigidity, MAGNETOCOSMICS accepts user defined latitude and longitude coordinates, altitude in the atmosphere, and direction of the primary particle that is entering the atmosphere relative to the geographic coordinates. The simulations calculate cutoff rigidity, the location dependent quantity that determines the minimum energy cosmic ray that can be observed at the earth's surface.

In our simulations, we used the Tsyganenko 89 [7] model for the external magnetic field and the IGRF 10 [8] model for the internal magnetic field. The atmosphere is defined to be $20 \mathrm{~km}$ above sea level. Simulated protons arrive at $90^{\circ}$ South latitude and $0^{\circ}$ longitude from a range of zenith and azimuth directions. This was done for the years 1969 and 2005 to be able to compare our results to [9]. After a preliminary scan across a wide range of zenith and azimuth, attention was focused only on larger zenith angles that showed significant increases in rigidity cutoffs. Beginning in 1965, in five year intervals, we calculated the cutoff rigidity for zenith angles $75^{\circ}$ and above with the full range of azimuth. Then, keeping 2005 as our reference year, we calculated the difference in rigidity cutoffs for each year simulated.

PLANETOCOSMICS [10] is a GEANT4 based simulation that transports particles through the earth's atmosphere. It includes effects due to the geomagnetic field. It also computes the flux of particles that reach a detector at user defined altitude and atmospheric depth.

Keeping the same definition for the altitude of the atmosphere, external field, internal field, date, and time; we simulated the flux of protons in 1965, 1975, 1985, 1995, and 2005 for the four zenith-azimuth combinations that corresponded to the greatest increases in cutoff rigidity. We focused on large values of rigidity because that's where the largest increases occurred. Neutron monitors observe cosmic rays from above the cutoffs, so it is worthwhile to examine that space. Ten thousand galactic cosmic rays, limited in our simulation to only protons, hit the atmosphere and produced a cascade of secondary particles. The atmospheric model used was NRLMSISE-00 [11]. To focus only on rigidities that could contribute to differences in neutron monitor rates, the energies of the protons ranged from the cutoff of 1965, 1975, 1985, or 1995 to the cutoff in 2005, as determined from the first part of this study. The protons were simulated to arrive above the South Pole. 2010 was left out of this part of the study because the change in rigidity across different incident directions showed increases in some bins and decreases in others, and excluding one year would not impact our analysis. The energy distribution of protons produced in the simulations 


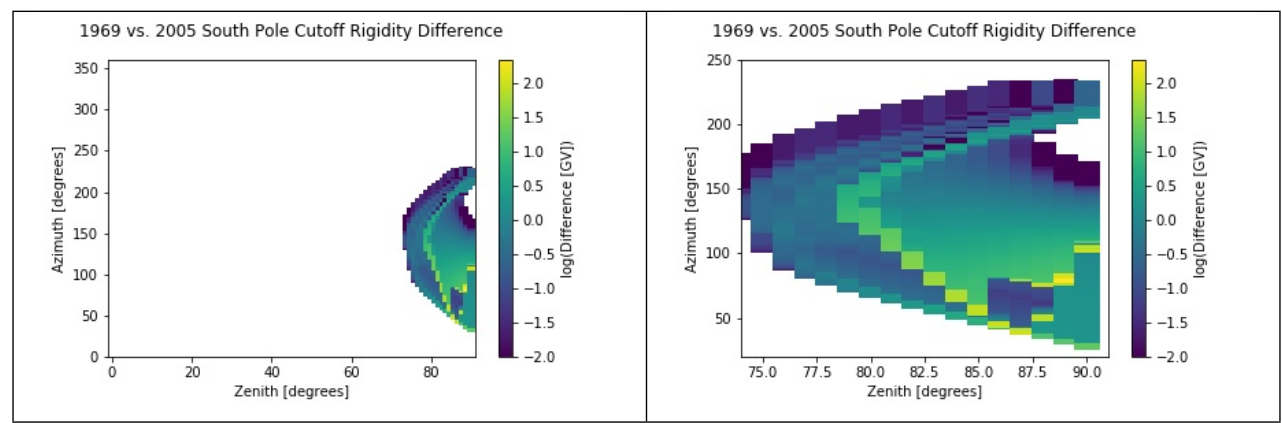

Figure 2: Difference in rigidity cutoffs, with respect to 2005, for the year 1969. It can be seen that there are increases in cutoff rigidity for higher zenith angles.

followed a spectral index of 2.65 [10] [12] [13] with the solar modulation parameter set to $500 \mathrm{MV}$, roughly the average over a solar cycle. We recorded the flux of neutrons arriving at an altitude of $3 \mathrm{~km}$, the altitude of SOPO on the glacial ice, with energies between $1.0 \mathrm{e}-04 \mathrm{MeV}$ and $1.0 \mathrm{e}+03 \mathrm{MeV}$.

\section{Results}

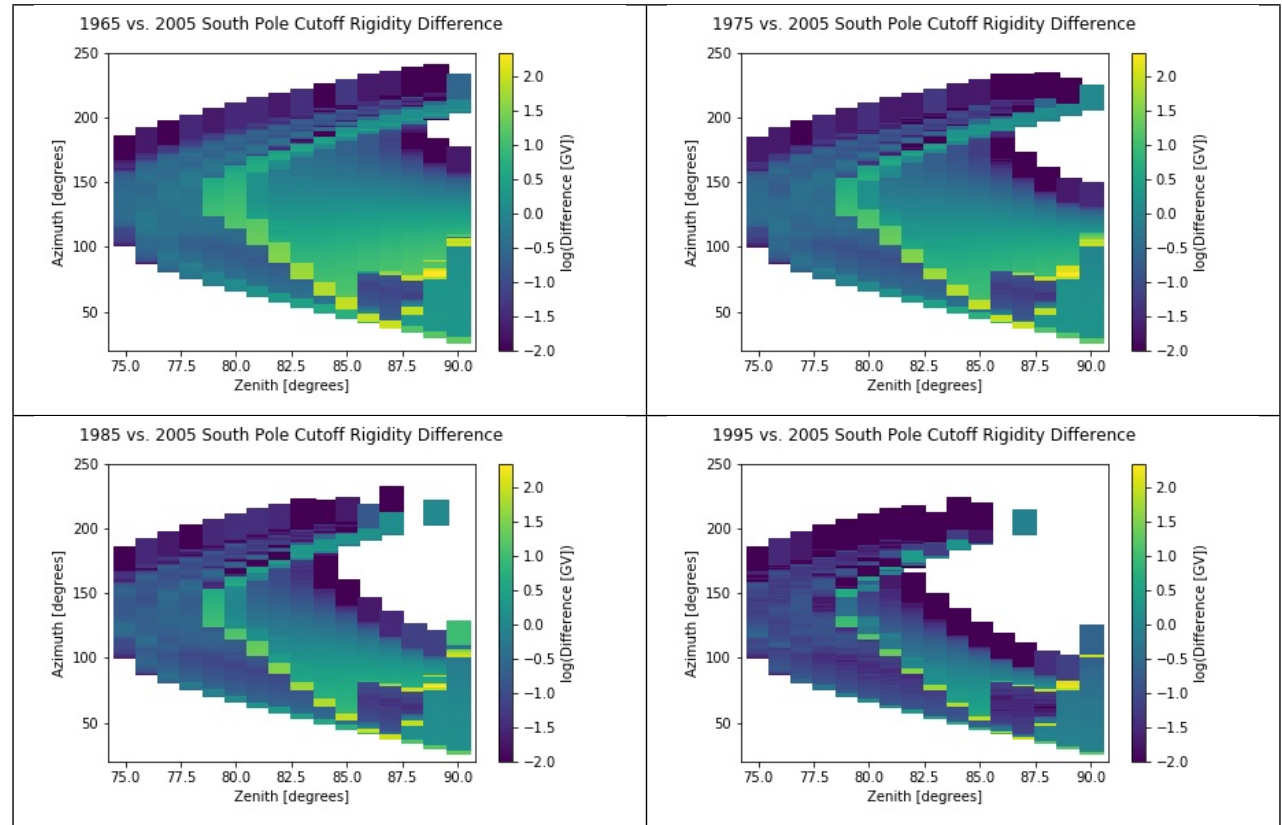

Figure 3: Difference in rigidity cutoffs, with respect to 2005, for years 1965, 1975, 1985, and 1995. There are regions of small azimuth bands at high zenith where the cutoffs increase significantly, and these temporal increases are gradual.

\subsection{Geomagnetic Cutoff Evolution}

Figures 2 and 3 show the main results on the evolution of geomagnetic cutoffs. In Figure 2, the full range of difference in rigidity cutoffs between 1969 and 2005 is shown. Analyzing more years 


\begin{tabular}{|c|c|c|c|c|c|c|}
\hline $\begin{array}{c}\text { Zenith } \\
\text { [degrees] }\end{array}$ & $\begin{array}{c}\text { Azimuth } \\
\text { [degrees] }\end{array}$ & $\mathbf{1 9 6 5}$ & $\mathbf{1 9 7 0}$ & $\mathbf{1 9 7 5}$ & $\mathbf{1 9 8 0}$ & $\mathbf{1 9 8 5}$ \\
\hline 89 & 87 & 23.51 & 23.51 & 23.95 & 24.11 & 24.31 \\
90 & 112 & 23.78 & 23.78 & 24.21 & 24.36 & 24.53 \\
88 & 84 & 99.87 & 99.88 & 99.88 & 99.88 & 99.89 \\
87 & 52 & 0.05 & 98.56 & 98.69 & 98.83 & 99.00 \\
\hline & & $\mathbf{1 9 9 0}$ & $\mathbf{1 9 9 5}$ & $\mathbf{2 0 0 0}$ & $\mathbf{2 0 0 5}$ & $\mathbf{2 0 1 0}$ \\
\hline 89 & 87 & 24.55 & 24.84 & 244.85 & 244.69 & 243.98 \\
90 & 112 & 24.70 & 24.88 & 99.07 & 147.98 & 146.32 \\
88 & 84 & 99.92 & 99.94 & 99.96 & 203.38 & 203.19 \\
87 & 52 & 99.12 & 99.16 & 99.20 & 99.23 & 99.27 \\
\hline
\end{tabular}

Table 1: Calculated cutoff rigidities, in GV, throughout the years for the zenith and azimuth combinations corresponding to the largest cutoff rigidity increases between 1965 and 2005. Simulations are run on December $12^{\text {th }}$ at 10 a.m. for each year.

in the regions that experienced large increases, each panel in Figure 3 shows the differences in cutoff rigidity at different zenith and azimuth, in GV, with respect to 2005. While for most incident directions the change in rigidity is negligible over the time period shown, there are small azimuth bands at high zenith where the rigidity increases significantly. The shapes of the bands are likely due to the viewing direction at the South Pole.

The numerical values of the calculated rigidities, along with their differences are displayed in Table 1. While other studies have corrected any cutoff rigidity values above $40 \mathrm{GV}$ to equal $40 \mathrm{GV}$ [9], we have kept the values as they are. In descending order, the four zenith-azimuth combinations corresponding to the greatest increases in cutoff rigidity are: 89 degrees zenith, 87 degrees azimuth; 90 degrees zenith, 112 degrees azimuth; 88 degrees zenith, 84 degrees azimuth; 87 degrees zenith, 52 degrees azimuth.

\subsection{Particles at the Surface}

The flux of neutrons reaching the surface for the four greatest increases in cutoff rigidities are given in Tables 2 and 3. No cut is made on the location of the neutrons at the Polar surface. Table 2 shows the theoretical flux of neutrons that would have reached the surface of the earth for each of the chosen zenith-azimuth combinations during the years 1965, 1975, 1985, and 1995. Table 3 uses the same energy ranges and conditions for simulating primary particles, except is run in the year 2005. Table 3 shows the theoretical flux that we would have seen in 2005 if it were not due to the increase in geomagnetic cutoffs.

The non-zero entries in the Normalized Flux column in Table 3 represent neutrons that would reach $3 \mathrm{~km}$ above the surface at the South Pole if the geomagnetic cutoff had not changed. In the same column in Table 2, the non-zero entries indicate that neutrons produced under the simulated conditions were reaching the glacial ice of the Polar surface before the increases in cutoffs. 


\begin{tabular}{|c|c|c|c|c|c|}
\hline $\begin{array}{c}\text { Zenith } \\
\text { [degrees] }\end{array}$ & $\begin{array}{l}\text { Azimuth } \\
\text { [degrees] }\end{array}$ & Year & $\begin{array}{c}\text { E }_{\min } \text { Primary } \\
{[\mathrm{GeV}]}\end{array}$ & $\begin{array}{c}\text { E }_{\text {max }} \text { Primary } \\
{[\mathrm{GeV}]}\end{array}$ & $\begin{array}{c}\text { Normalized Flux } \\
{\left[\text { neutrons } / \mathbf{c m}^{2} / \mathbf{s} / \text { primary flux] }\right.}\end{array}$ \\
\hline \multirow[t]{4}{*}{89} & \multirow[t]{4}{*}{87} & 1965 & 22.59 & 243.75 & $7.097 \mathrm{e}-05$ \\
\hline & & 1975 & 23.03 & 243.75 & $9.099 \mathrm{e}-05$ \\
\hline & & 1985 & 23.39 & 243.75 & $5.200 \mathrm{e}-05$ \\
\hline & & 1995 & 23.92 & 243.75 & $6.586 \mathrm{e}-05$ \\
\hline \multirow[t]{4}{*}{90} & \multirow[t]{4}{*}{112} & 1965 & 22.86 & 147.04 & $8.665 \mathrm{e}-06$ \\
\hline & & 1975 & 23.29 & 147.04 & $1.050 \mathrm{e}-06$ \\
\hline & & 1985 & 23.61 & 147.04 & $6.155 \mathrm{e}-06$ \\
\hline & & 1995 & 23.95 & 147.04 & $1.002 \mathrm{e}-06$ \\
\hline \multirow[t]{4}{*}{88} & \multirow[t]{4}{*}{84} & 1965 & 98.94 & 202.44 & $8.197 \mathrm{e}-06$ \\
\hline & & 1975 & 98.95 & 202.44 & $9.837 \mathrm{e}-06$ \\
\hline & & 1985 & 98.96 & 202.44 & $9.306 \mathrm{e}-06$ \\
\hline & & 1995 & 99.01 & 202.44 & $7.203 \mathrm{e}-06$ \\
\hline \multirow[t]{4}{*}{87} & \multirow[t]{4}{*}{52} & 1965 & $1.33 \mathrm{e}-03$ & 98.30 & $1.116 \mathrm{e}-03$ \\
\hline & & 1975 & 97.76 & 98.30 & $1.492 \mathrm{e}-07$ \\
\hline & & 1985 & 98.07 & 98.30 & $5.232 \mathrm{e}-08$ \\
\hline & & 1995 & 98.23 & 98.30 & $1.282 \mathrm{e}-08$ \\
\hline
\end{tabular}

Table 2: Values showing the normalized flux of neutrons arriving at the surface of the earth at $3 \mathrm{~km}$ altitude. These secondary neutrons are products of primary protons approaching the South Pole, defined in our simulations to have energies between the cutoff in 1965, 1975, 1985, or 1995 and the cutoff in the reference year 2005. Keeping consistent with the cutoff rigidity simulations, the date and time of the simulations is December $12^{\text {th }}$, at 10:00 a.m.

\section{Summary}

While cutoff rigidity depends on direction, typically the rigidity reported for a location refers only to the vertical cutoff rigidity. Our results demonstrate that between 1969 and 2005, an increase in cutoff rigidities could have occurred at high zenith angles for certain azimuth. Some of these increases are quite significant, meaning that particles arriving from those directions now require much greater energy to pass through the earth's geomagnetic field compared to in the past. Table 2 shows the flux of neutrons on the surface, $3 \mathrm{~km}$ height, produced by cosmic ray protons arriving at $20 \mathrm{~km}$ in the upper atmosphere at $90^{\circ}$ South latitude, and in the energy ranges suggested by the change in rigidity. No cut was made on a specific location on the surface. The fluxes represent neutrons that would arrive at the Polar surface had the cutoffs not increased in the given directions. This further supports the idea that evolving geomagnetic effects contribute to the declining rates at the South Pole. A full simulation of SOPO's responses to these neutrons will be examined in future work. It will also be worthwhile to explore cosmic rays arriving from directions where the rigidity did not change as much, but where it might better overlap with the region of greatest sensitivity to the neutron monitors. This may reveal a larger deficit of neutrons. 


\begin{tabular}{|c|c|c|c|c|c|}
\hline $\begin{array}{c}\text { Zenith } \\
\text { [degrees] }\end{array}$ & $\begin{array}{l}\text { Azimuth } \\
\text { [degrees] }\end{array}$ & Year & $\begin{array}{c}\text { Emin }_{\text {min }} \text { Primary } \\
{[\mathrm{GeV}]}\end{array}$ & $\begin{array}{c}\text { E }_{\text {max }} \text { Primary } \\
{[\mathrm{GeV}]}\end{array}$ & $\begin{array}{c}\text { Normalized Flux } \\
\text { [neutrons } / \mathbf{c m}^{2} / \mathrm{s} / \text { primary flux] }\end{array}$ \\
\hline \multirow[t]{4}{*}{89} & \multirow[t]{4}{*}{87} & 2005 & 22.59 & 243.75 & $9.517 \mathrm{e}-05$ \\
\hline & & 2005 & 23.03 & 243.75 & $6.111 \mathrm{e}-05$ \\
\hline & & 2005 & 23.39 & 243.75 & $8.690 \mathrm{e}-05$ \\
\hline & & 2005 & 23.92 & 243.75 & $9.211 \mathrm{e}-05$ \\
\hline \multirow[t]{4}{*}{90} & \multirow[t]{4}{*}{112} & 2005 & 22.86 & 147.04 & $7.581 \mathrm{e}-06$ \\
\hline & & 2005 & 23.29 & 147.04 & $1.365 \mathrm{e}-05$ \\
\hline & & 2005 & 23.61 & 147.04 & $2.054 \mathrm{e}-06$ \\
\hline & & 2005 & 23.95 & 147.04 & $1.002 \mathrm{e}-06$ \\
\hline \multirow[t]{4}{*}{88} & \multirow[t]{4}{*}{84} & 2005 & 98.94 & 202.44 & $7.452 \mathrm{e}-06$ \\
\hline & & 2005 & 98.95 & 202.44 & $7.891 \mathrm{e}-06$ \\
\hline & & 2005 & 98.96 & 202.44 & $7.757 \mathrm{e}-06$ \\
\hline & & 2005 & 99.01 & 202.44 & $1.121 \mathrm{e}-05$ \\
\hline \multirow[t]{4}{*}{87} & \multirow[t]{4}{*}{52} & 2005 & $1.33 \mathrm{e}-03$ & 98.30 & $2.073 \mathrm{e}-03$ \\
\hline & & 2005 & 97.76 & 98.30 & $1.414 \mathrm{e}-07$ \\
\hline & & 2005 & 98.07 & 98.30 & $4.366 \mathrm{e}-08$ \\
\hline & & 2005 & 98.23 & 98.30 & $1.605 \mathrm{e}-08$ \\
\hline
\end{tabular}

Table 3: Values showing the normalized flux of neutrons arriving at the surface of the earth at $3 \mathrm{~km}$ altitude. These secondary neutrons are products of primary protons approaching the South Pole, defined in our simulations to have energies between the cutoff in 1965, 1975, 1985, or 1995 and the cutoff in the reference year 2005. Keeping consistent with the cutoff rigidity simulations, the date and time of the simulations is December 12, 2005 at 10 a.m.

\section{Acknowledgements}

This work was supported in parts by NSF Integrative Activities in Physics, REU Site: Research In Neutrino Astrophysics at the University of Wisconsin-River Falls, Awards 1460752 and 1757517, and NSF OPP, Antarctic Education, ATE Award 1341312. We thank Dr. Waraporn Nuntiyakul, Chiang Mai University, for her helpful advice during this project; Dr. Philip von Doetinchem, University of Hawaii, for access to his modified and updated PLANETOCOSMICS code; and Dr. Rolf Buetikofer, University of Bern, for access and permission to use both MAGNETOCSOMICS and PLANETOCOSMICS. We thank Dr. Paul Evenson for including discussions of this project in the weekly neutron monitor working group conference call, and UWRF physics for providing the facilities needed to work on this project.

\section{References}

[1] John A. Simpson. The cosmic ray nucleonic component: The invention and scientific uses of the neutron monitor - (keynote lecture). Space Science Reviews, 93:11-32, 042012.

[2] Paul Evenson, John Bieber, John Clem, and Roger Pyle. South pole neutron monitor lives again. Proceedings of the 32nd International Cosmic Ray Conference, ICRC 2011, 11, 012011. 
[3] J. W. Bieber, J. Clem, D. Desilets, P. Evenson, D. Lal, C. Lopate, and R. Pyle. Long-term decline of south pole neutron rates. Journal of Geophysical Research: Space Physics, 112(A12).

[4] John Bieber, John Clem, Paul Evenson, Suyeon Oh, and Roger Pyle. Continued decline of south pole neutron monitor counting rate. Journal of Geophysical Research: Space Physics, 118, 112013.

[5] Laurent Desorgher. Magnetocosmics. cosray.unibe.ch/ laurent/magnetocosmics, 2006. Accessed 2019-07-04.

[6] S. Agostinelli et. al. Geant4-a simulation toolkit. Nuclear Instruments and Methods in Physics Research Section A: Accelerators, Spectrometers, Detectors and Associated Equipment, 506(3):250 303, 2003.

[7] N.A. Tsyganenko. A magnetospheric magnetic field model with a warped tail current sheet. Planetary and Space Science, 37(1):5-20, 1989.

[8] Susan Macmillan and Stefan Maus. International geomagnetic reference field - the tenth generation. Earth, Planets, and Space, 57(12):1135-1140, 2005.

[9] John Clem. South pole neutron monitor sensitivity to geomagnetic field variations. Proc. 34th International Cosmic Ray Conference (The Hague) 2015, page 143, 082015.

[10] Laurent Desorgher. Planetocosmics. cosray •unibe.ch/ laurent/planetocosmics/, 2006. Accessed 2019-07-04.

[11] J. M. Picone, A. E. Hedin, D. P. Drob, and A. C. Aikin. Nrlmsise-00 empirical model of the atmosphere: Statistical comparisons and scientific issues. Journal of Geophysical Research: Space Physics, 107(A12):SIA 15-1-SIA 15-16, 2002.

[12] L. A. Fisk and W. I. Axford. Solar modulation of galactic cosmic rays, 1. Journal of Geophysical Research, 74(21):4973-4986, 1969.

[13] M. Garcia-Munoz, G. M. Mason, and J. A. Simpson. The anomalous ${ }^{4} \mathrm{He}$ component in the cosmic-ray spectrum at $50 \mathrm{MeV}$ per nucleon during 12972 - 1974. , 202:265-275, Nov 1975. 